\title{
Design and Application of a Dual Processor Digital Control System for Ultra-High Frequency Pulsed Gas Tungsten Arc Welding
}

\author{
Wei $\mathrm{Li}^{1}$, Gaochong $\mathrm{Lv}^{1}$, Bojin $\mathrm{Qi}^{2}$, Songtao Huang ${ }^{3}$, Huina Jiang ${ }^{1^{*}}$ \\ ${ }^{1}$ Information Engineering College, Beijing Institute of Petrochemical Technology, Beijing 102617, China \\ ${ }^{2}$ School of Mechanical Engineering and Automation, Beihang University, Beijing, 100191, China \\ ${ }^{3}$ Mechanical Engineering College, Beijing Institute of Petrochemical Technology, Beijing, 102617, China
}

Corresponding Author Email: Jianghuina@bipt.edu.cn

https://doi.org/10.18280/jesa.530214

Received: 17 December 2019

Accepted: 10 February 2020

\section{Keywords:}

dual processor, digital control, ultra-highfrequency pulse modulation, gas tungsten arc welding $($ GTAW)

\begin{abstract}
Ultra-high-frequency pulse modulation is an innovative and effective means of arc regulation. However, the application of this technique in gas tungsten arc welding (GTAW) faces several problems. To solve these problems, this paper selects a main circuit topology for the welding power sources, and designs a dual processor digital control system, in which the pulse width modulation (PWM) signals are generated collaboratively by a digital signal processor (DSP) and a complex programmable logic device (CPLD). Through simulations and experiments, it is learned that our control system could output ultra-high pulsed GTAW current of over 100A, and realize a fast current conversion rate. Besides, multiple welding modes could be realized easily without changing the system design or electrical connection. The proposed control system effectively automates ultrahigh-frequency pulsed gas tungsten arc welding (UHFP-GTAW), and promotes the engineering application of ultra-high-frequency pulse modulation.
\end{abstract}

\section{INTRODUCTION}

Introducing pulse energy to the welding process, pulsed gas tungsten arc welding (P-GTAW) effectively controls the state of arc energy, and thus promotes the weld quality and joint performance [1-4]. Relevant studies have shown that, the pulsed current modulation applied to welding arc can effectively improve arc pressure [5-7]. Once the current pulse reaches a sufficiently high frequency, the welding arc will produce an obvious high-frequency effect: the welding arc will be more stable, and the liquid metal will crystalize better in the molten pool [8-10]. As a result, high-frequency pulse modulation has been successfully applied to optimize the welding of various metals and the properties of weld joints.

Pulse modulation in the GTAW has attracted much attention from scholars around the world [11-16]. However, the pulse frequency rarely exceeds $1 \mathrm{kHz}$ and the current amplitude seldom surpasses $100 \mathrm{~A}$ in the existing studies, under the constraints of circuit technology and control system scheme. The limited pulse frequency and low current restrict the role of pulse modulation in arc regulation. In the past decade, Beihang University probed deep into ultra-high-frequency $(>20 \mathrm{kHz})$ pulse modulation: the welding platform was built based on new main circuit topology and control method, and used to perform various types of welding of typical alloys under ultra-high-frequency pulse modulation, such as ultrahigh-frequency pulsed gas tungsten arc welding (UHFPGTAW) of aluminum alloy and ultra-high-frequency pulsed variable polarity gas tungsten arc welding (UHFPVP-GTAW) of titanium alloy and stainless steel. The results show that ultra-high-frequency pulse modulation effectively improves weld quality and joint performance $[17,18]$. Thanks to ultrahigh-frequency pulse modulation, the inherent defects (e.g. pores and cracks) of welding methods are reduced and even eliminated, and the weld quality could reach the strict requirement for high-precision fields like aerospace. Therefore, it is very meaningful to apply this modulation technique in various engineering fields.

Nonetheless, the proliferation of ultra-high-frequency pulse modulation is bottlenecked by the defects with the current welding platform. For instance, most commercial welding power sources demand different welding methods be realized on the same welding device, without changing the electrical connection. But the current welding platform needs to change the electrical wiring of the hardware system, before switching to another welding method. What is worse, the current welding platform, unable to change the welding mode flexibly, cannot achieve automatic welding. To complete a welding experiment, manual intervention is needed in different stages of the welding process.

To solve these defects, this paper puts forward a reasonable dual processor digital control system that facilitates the application of ultra-high-frequency pulse modulation. Firstly, the main circuit topology of the system was detailed. Next, the dual processor control was explained, which realizes the fast current conversion required by the topology. Then, the generation scheme of pulse width modulation (PWM) signals in the system was proved feasible through simulation. Finally, the proposed digital control system was applied to the welding of various alloys. The results fully demonstrate the effectiveness of our system.

\section{MAIN CIRCUIT TOPOLOGY}

Figure 1 shows the main circuit topology of our system, 
which achieves fast current conversion based on parallel and concatenated mode [19]. The composite current conversion system consists of three parallel power sources and three levels of cascade current conversion modules. The power sources and current conversion modules are all independent of each other, such that each characteristic parameter of current could be controlled precisely and separately in the welding process. The components of the main circuit are described as follows:

The pre-circuit encompasses the first, the second and the third constant current sources in parallel, which provide base current $I_{\mathrm{I}}$, positive peak current $I_{\mathrm{II}}$, and reverse peak current $I_{\mathrm{III}}$ respectively. The middle circuit is composed of positive and negative high-frequency pulsed switching circuits, which convert the constant current from the pre-circuit into ultrahigh-frequency pulsed current with fast change rate.

The positive and negative pulsed currents $I_{\mathrm{p}+}$ and $I_{\mathrm{p}-}$ can be superimposed on the first constant current source of the precircuit in the high- and low-level stages of the pulse control signal, respectively. The output effect of ultra-high-frequency pulsed current $I_{\mathrm{p}^{+}}$depends on the fast alternation actions of two insualted gate bipolar transistors (IGBTs) $\mathrm{VT}_{5}$ and $\mathrm{VT}_{6}$ at a certain frequency. Similarly, the frequency of negative pulsed

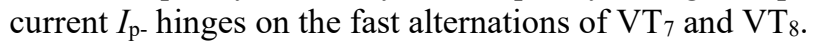

The post-circuit shown consists of a full-bridge current polarity conversion circuit and an auxiliary absorption circuit. As per the welding requirements, the full-bridge current polarity conversion circuit conducts polarity conversion of output current $I_{\mathrm{cp}}$ from the middle circuit. The composite current $I_{\mathrm{vp}}$ from the post-circuit is controlled by PWM signals, and outputted in different forms: traditional variable polarity current, ultra-high-frequency pulsed current, and ultra-highfrequency pulsed variable polarity current.

Note that the composite current is transferred to the electrode and the workpiece via ordinary cables. The change rate of the composite current is as fast as $50 \mathrm{~A} / \mu \mathrm{s}$, under proper protection and absorption measures. In addition, if current polarity conversion is conducted, the composite current can achieve zero-crossing without dead-time, and current polarity can be converted at $50 \mathrm{~A} / \mu \mathrm{s}$. In this case, the welding arc will remain stable without any additional stabilization measures.

Based on the main circuit topology, the power sources could output various currents for various welding modes through sequential and logical adjustment of the PWM signals PWM18 that switches IGBTs $\mathrm{VT}_{1}-\mathrm{VT}_{8}$, eliminating the need to change the hardware design.

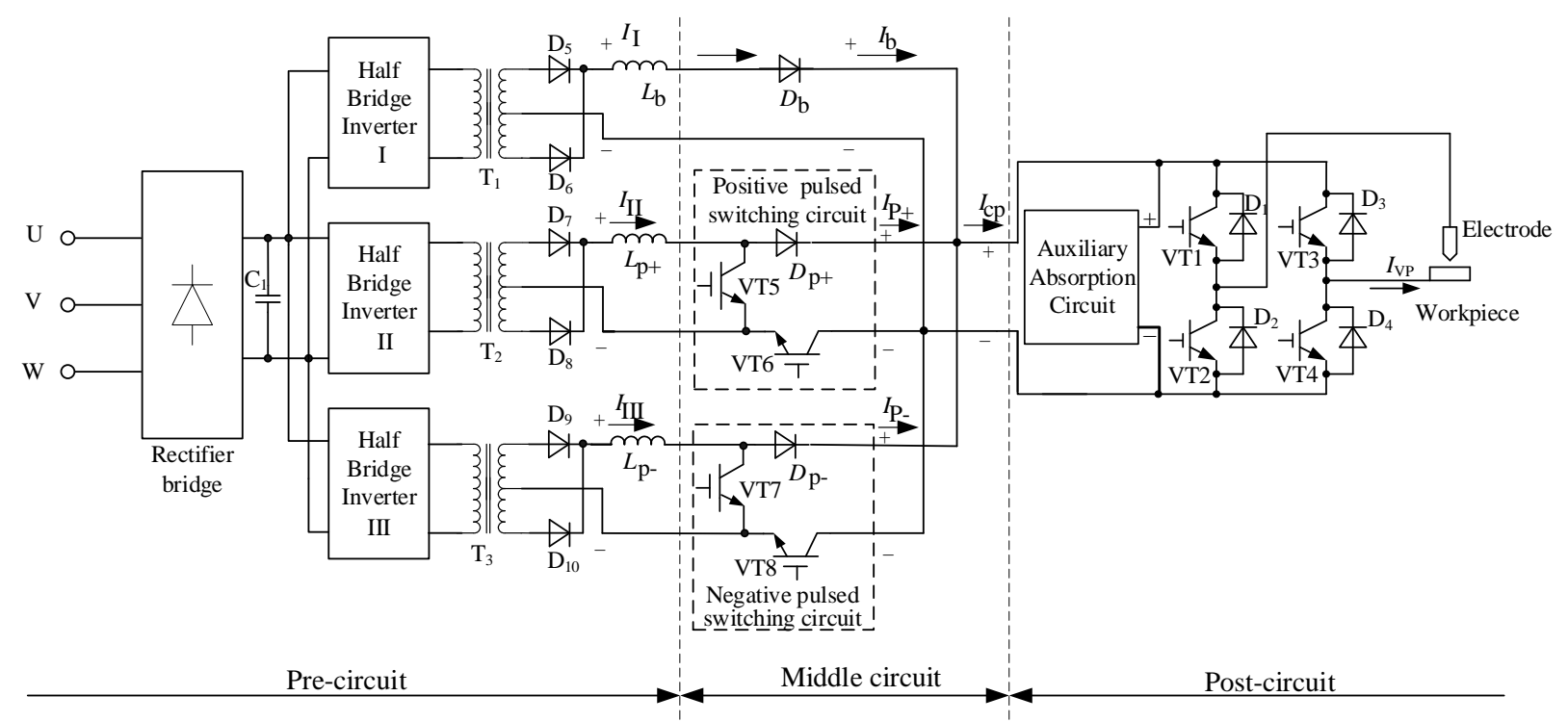

Figure 1. The main circuit topology

\section{DESIGN OF DIGITAL CONTROL SYSTEM}

\subsection{Dual processor control}

As shown in Figure 2, our digital control system has two processors: a digital signal processor (DSP) dsPIC30F6012A, and a micro-controller PIC24FJ256GB106. The main functions of the DSP include digital setting, adjustment of constant current source, control of high-frequency arc ignition, and unified regulation of welding parameters. The main functions of the micro-controller include human-computer interaction (HCI), USB storage, and invocation of welding parameters.

The limited amount of communication data between the two processors are exchanged in the RS232 serial communication mode. In addition, the DSP, in association with a complex programmable logic device (CPLD) EPM3128ATC100, generate required PWM control signals of IGBTs.

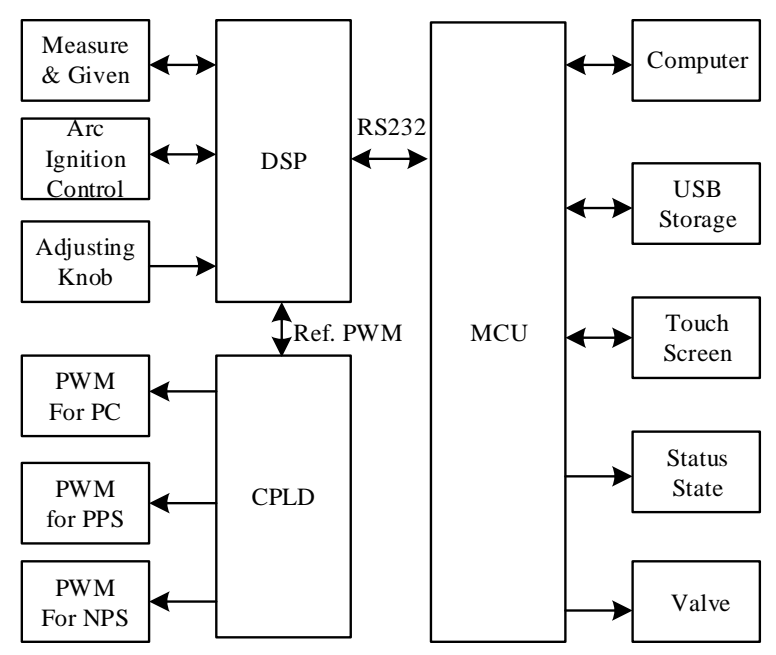

Figure 2. The dual processor control 


\subsection{Digital PWM output}

For both the full-bridge current polarity conversion circuit and the ultra-high-frequency pulsed switching circuits, the PWM signals that control the switch state of IGBTs $\mathrm{VT}_{1}-\mathrm{VT}_{8}$ are all hybrid pulsed signals, featuring complex sequential and logical relations.

If all the PWM signals are generated by the DSP alone, it will be difficult to consider other real-time tasks ensuring the reliable output of PWM signals, despite that complex PWM signals can be achieved by frequently rewriting relevant registers in interrupt program of timers.

Thanks to its flexible logic combination capability, the CPLD was coupled with the DSP to generate the digital PWM signals in our system. As shown in Figure 3, the DSP is responsible for generating two pairs of complementary PWM signals with dead-time function, namely, variable polarity PWM and high-frequency pulsed PWM. Referring to the two pairs of PWM signals, the CPLD combines variable polarity PWM signal with high-frequency pulsed PWM signal, by the logic dependent on the mode control signals represented by CTR in simulation diagram and later description, and generates three pairs of PWM signals, which control fullbridge polarity conversion circuit, positive high-frequency pulsed switching circuit, and negative high-frequency pulsed switching circuit, respectively.

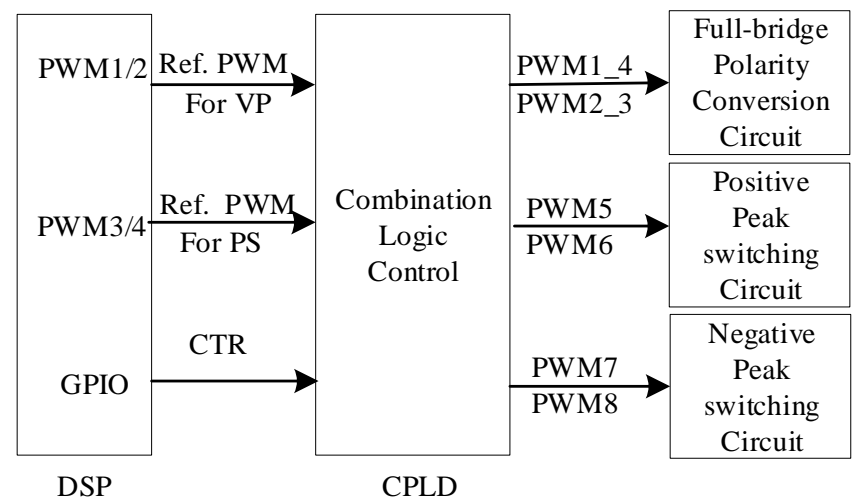

Figure 3. DSP + CPLD geenration of digital PWM sginals

\subsection{PWM generation software}

The PWM signals for reference polarity conversion are generated by the PWM generator in the motor PWM control module of the DSP. This module provides up to four pairs of PWM generators. The frequencies of these generators must be kept consistent, rather than be adjusted separately. This is because the frequency of each generator differs from that of reference polarity conversion circuit. In the reference pulsed switching circuit, the PWM signals are generated by two comparison modules in the DSP through software programming.

After powered on, the DSP program automatically generates all the reference PWM signals with given frequency and duty ratio by simple initialization. There is no need for any software intervention of the PWM output, unless the user changes frequency or duty ratio through the HCI. To improve the current conversion rate, all the complementary PWM signals are designed to have a common conduction time, rather than a dead zone.

The final PWM signals to control the IGBTs are generated by the CPLD, according to the input/output (I/O) status of the CTR representing different welding modes. The CTR consists of three digital I/Os of the DSP, with a binary value falling within $[000,111]$. Table 1 lists the binary value of the MCS for each welding mode. Among them, CTR $=001$ stands for positive pulsed modulation mode (PP mode), 010 represents negative pulsed modulation mode (NP mode), etc.

The PWM outputs of PP model was simulated by design software Quartus II. During the simulation, the frequencies of PWMP1 and PWMP2, a pair of complementary PWM signals provided by the DSP for reference polarity conversion, were set to $1 \mathrm{kHz}$; the frequencies of PWMH1 and PWMH2, a pair of complementary PWM signals provided by the DSP for reference pulsed switching, were set to $20 \mathrm{kHz}$. The simulation results are presented in Figure 4.

Table 1. The binary values of the MCS for each welding mode

\begin{tabular}{cccc}
\hline CTR & Mode & CTR & Mode \\
\hline 000 & Output-off & 100 & Common VP \\
001 & PP modulation & 101 & Pulsed DC \\
010 & NP modulation & 110 & DCEP \\
011 & BP modulation & 111 & DCEN \\
\hline
\end{tabular}

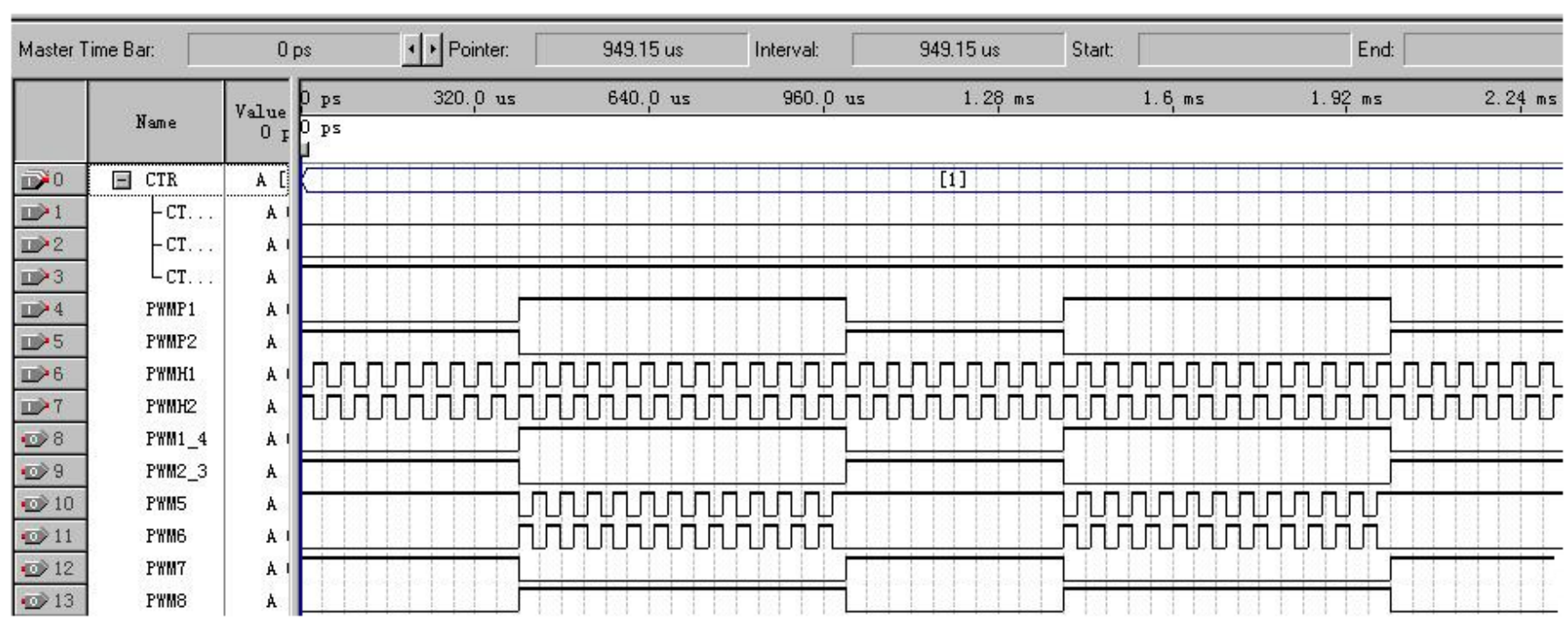

Figure 4. The complex modulation PWM outputs under PP welding mode 
As shown in Figure 4, the output signals PWM1 4 and PWM2 3 control $\mathrm{VT}_{1} / \mathrm{VT}_{4}$ and $\mathrm{VT}_{2} / \mathrm{VT}_{3}$ in the full-bridge polarity switching circuit, respectively. PWM5 and PWM6 control $\mathrm{VT}_{5}$ and $\mathrm{VT}_{6}$ of the forward peak switching circuit, respectively. PWM7 and PWM8 control $\mathrm{VT}_{7}$ and $\mathrm{VT}_{8}$ of the reverse peak switching circuit, respectively.

\section{EXPERIMENTAL VERIFICATION}

\subsection{Realization of UHFPVP-GTAW current}

With aluminum alloy plate as the test material, the characteristic parameters of welding current were set on the HCI of touch screen: base current, 100A; forward peak current, $100 \mathrm{~A}$; reverse peak current, $80 \mathrm{~A}$; variable polarity frequency, $500 \mathrm{~Hz}$; variable polarity duty ratio, $80 \%$; pulse frequency, $20 \mathrm{kHz}$; pulse duty ratio, $20 \%$. Under the welding mode of BP, the output waveform of UHFPVP-GTAW current was measured by a Hall effect current sensor and a digital oscilloscope (Figure 5(a)).

\subsection{Realization of UHFP-GTAW current}

The UHFP-GTAW process was adopted to weld titanium alloy. The binary value of the CTR was set to 101, so that the power source produces ultra-high-frequency pulsed current without polarity conversion. Then, the pulsed frequency and pulsed duty ratio were set to $20 \mathrm{kHz}$ and $80 \%$, respectively; the base current, forward peak current, and reverse peak current were all configured as 100A. On this basis, the pulsed current of the UHFP-GTAW was captured (Figure 5(b)).

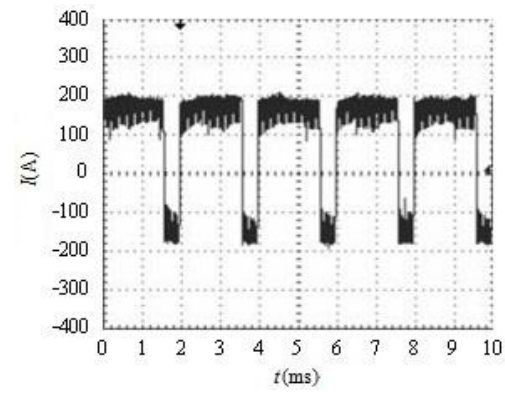

(a) UHFPVP-GTAW current in BP mode

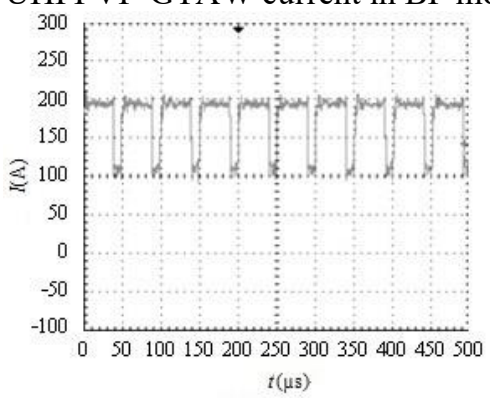

(b) UHFP-GTAW current

Figure 5. The output current waveforms

\subsection{Test on typical welding process}

During traditional arc welding, aluminum alloy and titanium alloy are prone to welding defects like gas pores. These defects seriously threat the mechanical properties of welded joints. Here, both aluminum alloy and titanium alloy are subjected to ultra-high-frequency pulsed welding on the welding platform regulated by our dual processor digital control system, aiming to verify the flexibility of welding mode switching and the applicability of ultra-high-frequency pulsed welding.

In aviation, the key components of equipment must be butt welded with two kinds of aluminum alloys (5A06 and 3A21). If the two dissimilar aluminum alloys are welded by traditional arc welding, the butt weld will have a high porosity, failing to meet the requirements on product design and performance.

To eliminate the pores in the weld seam, the UHFPVPGTAW process (Subsection 4.1) was selected to weld the test samples. The welding results (Figure 6) show that the well seam is well formed, the surface is bright, and no pore was observed.

The TC4 titanium alloy is a popular material in various fields, ranging from aerospace, transport, to biomedicine [20]. If the traditional GTAW is adopted to weld the TC4, the material must go through a complex cleaning process before welding, and be protected strictly in the course of welding. Otherwise, the weld seam will definitely carry porosity defect.

Here, a $1.5 \mathrm{~mm}$-thick TC4 plate is welded by the UHFPGTAW process (Subsection 4.2). Before welding, the specimen and the filler wires were wiped with acetone to remove the surface oil stains, and cleaned mechanically to rid the surface oxide film. The welding results (Figure 7) show that the weld seam is well formed, without any pores, although no protective measure was adopted in the UHFP-GTAW process.

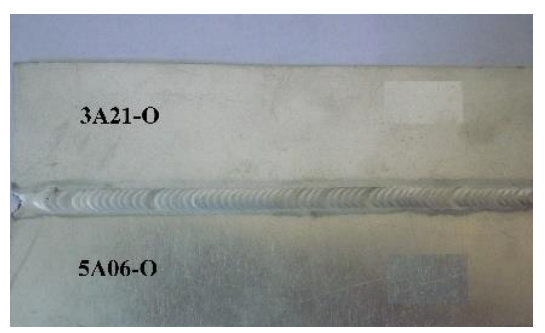

(a) Front view

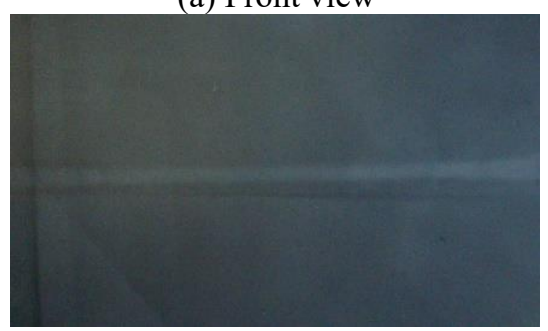

(b) Image of X-ray flaw detection

Figure 6. The weld quality of test samples

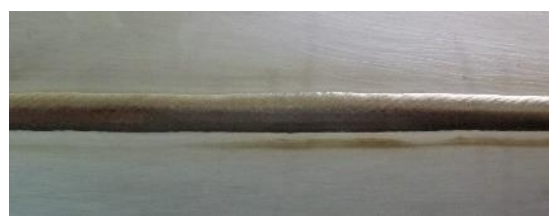

(a) Front view

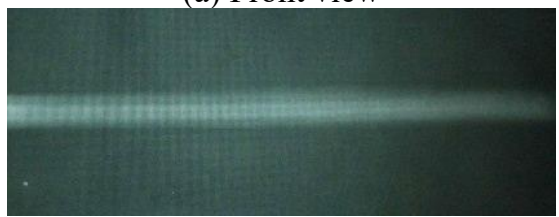

(b) Image of X-ray flaw detection

Figure 7. The weld quality of the TC4 plate 


\subsection{Discussion}

From the experimental results, it is learned that the welding arc takes the high-frequency compression effect, as the welding current is modulated by ultra-high-frequency pulse. The compression effect increases with the frequency. Compared with traditional GTAW process, the UHFP-GTAW process can compress welding arc obviously along radial direction and increase the stiffness of the arc. As a result, the welding arc becomes more stable, which promotes the weld quality.

The elimination of gas pores is attributable to two factors. First, the compression of welding arc reduces the surface area of molten pool, which in turn improves the gas protection effect. Then, it is less likely for gas pores to form in the molten pool. Second, the arc pressure is greatly increased by ultrahigh-frequency pulsed modulation. Under the high pressure, the welding arc digs deeper into the molten pool, making the liquid metal more fluid. In this case, the bubbles can escape to the surface of the molten pool more easily, reducing the probability of gas pores in the weld seam.

\section{CONCLUSIONS}

To automate the UHFP-GTAW, this paper designs a main circuit topology, which achieves fast current conversion based on parallel and concatenated mode. On this basis, a dualprocessor digital control system was designed for accurately control and separate regulation of the characteristic parameters of welding current.

The DSP and the CPLD were coupled to develop a digital PWM generation scheme. The CPLD is responsible for pulsed modulation, while the DSP only provides the reference PWM signals. The integrated scheme supports multiple modulation modes of ultra-high-frequency pulse, without changing hardware design or electrical connection.

Through welding experiments on titanium alloy and aluminum alloy, it is confirmed that ultra-high-frequency pulse modulation eliminates the gas pole defects better than the traditional GTAW.

\section{ACKNOWLEDGMENT}

This work was supported by the National Natural Science Foundation of China (Grant No.: 51675052). Our thanks go to Beijing Institute of Petrochemical Technology for its substantial supports to this work.

\section{REFERENCES}

[1] Manti, R., Dwivedi, D.K. (2007). Microstructure of Al$\mathrm{Mg}-\mathrm{Si}$ weld joints produced by pulse TIG welding. Materials and Manufacturing Processes, 22(1): 57-61. https://doi.org/10.1080/10426910601015923

[2] He, L., Yang, P., Li, L., Wu, M. (2014). The ultrasonic characteristics of high frequency modulated arc and its application in material processing. Ultrasonics, 54(8): 2178-2183. https://doi.org/10.1016/j.ultras.2014.06.003

[3] Balasubramanian, V., Ravisankar, V., Reddy, G.M. (2008). Effect of pulsed current welding on mechanical properties of high strength aluminum alloy. The
International Journal of Advanced Manufacturing Technology, 36(3-4): 254-262. https://doi.org/10.1007/s00170-006-0848-0

[4] Stoeckinger, G.R. (1973). Pulsed DC high frequency GTA welding of aluminum plate. Welding journal, 52(12): 558

[5] Qiu, L., Fan, C., Lin, S., Yang, C. (2007). Highfrequency pulse modulated variable polarity welding power and its arc pressure. Transactions-China Welding Institution, 28(11): 81-84.

[6] Cook, G.E., Eassa, H.E.D.E. (1985). The effect of highfrequency pulsing of a welding arc. IEEE Transactions on Industry Applications, (5): 1294-1299. https://doi.org/10.1109/TIA.1985.349557

[7] Yang, M., Li, L., Qi, B., Zheng, H. (2017). Arc force and shapes with high-frequency pulsed-arc welding. Science and Technology of Welding and Joining, 22(7): 580-586. https://doi.org/10.1080/13621718.2016.1277625

[8] Zhao, J.R., Li, Y.D. (1989). Control over arc of pulsed high frequency TIG welding and high frequency effect. Journal of Tianjin University, 3: 25-32.

[9] Yan, S.B., Song, Y.L., Zhang, J., Bai, L.L. (2012). The high frequency pulse effect and verification in the welding process for high-strength aluminum alloy. In Applied Mechanics and Materials, 130: 27-31. https://doi.org/10.4028/www.scientific.net/AMM.130134.27

[10] Morisada, Y., Fujii, H., Inagaki, F., Kamai, M. (2013). Development of high frequency tungsten inert gas welding method. Materials \& Design, 44: 12-16. https://doi.org/10.1016/j.matdes.2012.07.054

[11] Anbarasan, N., Jerome, S., Suresh, G., Oyyaravelu, R. (2019). Effect of Pulse Frequency on Microstructural and Corrosion Properties of Inconel 718 Gas Tungsten Arc Weldments. Transactions of the Indian Institute of Metals, 72(5): 1299-1311. https://doi.org/10.1007/s12666-019-01626-y

[12] Prakash, C., Singh, S., Singh, M., Gupta, M.K., Mia, M., Dhanda, A. (2019). Multi-objective parametric appraisal of pulsed current gas tungsten arc welding process by using hybrid optimization algorithms. The International Journal of Advanced Manufacturing Technology, 101(1 4): 1107-1123. https://doi.org/10.1007/s00170-0183017-3

[13] Subramani, P., Manikandan, M. (2019). Development of gas tungsten arc welding using current pulsing technique to preclude chromium carbide precipitation in aerospacegrade alloy 80A. International Journal of Minerals, Metallurgy, and Materials, 26(2): 210-221. https://doi.org/10.1007/s12613-019-1726-8

[14] Kumar, R., Anant, R., Ghosh, P.K., Kumar, A., Agrawal, B.P. (2016). Influence of PC-GTAW parameters on the microstructural and mechanical properties of thin AISI 1008 steel joints. Journal of Materials Engineering and Performance, $\quad$ 25(9): 3756-3765. https://doi.org/10.1007/s11665-016-2211-4

[15] Rahimi, A., Shamanian, M., Szpunar, J.A. (2020). Effect of pulsed current frequency on microstructure and mechanical properties of Gas-Tungsten-Arc-Welded Joints of UNS R30155. Journal of Materials Engineering and Performance, 29(4): 2635-2647. https://doi.org/10.1007/s11665-020-04752-7

[16] Balasubramanian, V., Ravisankar, V., Reddy, G.M. (2008). Effect of pulsed current welding on mechanical 
properties of high strength aluminum alloy. The International Journal of Advanced Manufacturing Technology, 36(3-4): 254-262. https://doi.org/10.1007/s00170-006-0848-0

[17] Cong, B.Q., Qi, B.J., Zhou, X.G., Luo, J. (2009). Influences of ultrasonic pulse square-wave current parameters on microstructures and mechanical properties of 2219 aluminum alloy weld joints. Acta Metall Sin, 45(9): 1057-1062. https://www.ams.org.cn/EN/Y2009/V45/I9/1057

[18] Yang, M.X., Qi, B.J., Cong, B.Q., Wang, L.X. (2012). Microstructure and properties of titanium alloys welds using ultrasonic frequency pulse GTAW. Transactions of the China Welding Institution, 33(6): 39-42.

[19] Qi, B.J., Cong, B.Q. (2008). A novel ultrafast-convert complex-pulse variable-polarity arc welding power topology. Transactions of the China Welding Institution, 29(11): 57-60, 116.

[20] Köse, C., Karaca, E. (2018). Effect of pre and post weld heat treatments on the microstructure and mechanical properties of fiber optic beam delivery system assisted robotic Nd: Yag Laser Welded Ti-6Al-4V Alloy. Archives of Metallurgy and Materials, 63(3): 1225-1233. https://doi.org/10.24425/123795 